ESAIM: PROCEEDINGS AND SURVEYS, September 2018, Vol. 62, p. 123-138

Muhammad DAUHOO, Laurent DUMAS, Pierre GABRIEL and Pauline LAFITTE

\title{
AN INTRODUCTION TO THE BASIC REPRODUCTION NUMBER IN MATHEMATICAL EPIDEMIOLOGY*
}

\author{
Antoine Perasso ${ }^{1}$
}

\begin{abstract}
This article introduces the notion of basic reproduction number $\mathcal{R}_{0}$ in mathematical epidemiology. After an historic reminder describing the steps leading to the statement of its mathematical definition, we explain the next-generation matrix method allowing its calculation in the case of epidemic models described by ordinary differential equations (ODEs). The article then focuses, through four ODEs examples and an infection load structured PDE model, on the usefulness of the $\mathcal{R}_{0}$ to address biological as well mathematical issues.
\end{abstract}

\section{INTRODUCTION}

The basic reproduction (or reproductive) number (or ratio) is a fundamental concept in epidemiology. It is a quantity, denoted $\mathcal{R}_{0}$, that represents the number of secondary infections resulting from a single primary infection into an otherwise susceptible population. Following the developpment of mathematical modeling approaches in epidemiology in the last century, $\mathcal{R}_{0}$ has become a key concept to prevent the apparition of epidemics, and one can find in the litterature a huge quantity of articles that mix mathematical modeling and $\mathcal{R}_{0}$ determination for the major part of the known diseases in the world. The goal of this article is to introduce the concept of basic reproduction number in mathematical epidemiology and to illustrate its usefulness through several examples, with biological as well as mathematical interest. We will mainly focus on ordinary differential equations (ODEs) models, but a short part of the article will be dedicated to an example of structured partial differential equations (PDEs) model, to enlighten the main difficulties that may arise when dealing with infinite dimensional problems.

The article is structured as follows : Section 1 is dedicated to the history of $\mathcal{R}_{0}$, explaining the different steps that allowed its mathematical definition as a threshold. Then we introduce in Section 2 the "next-generation matrix" method, usefull to define and calculate a $\mathcal{R}_{0}$ from ODEs models. Then Section 3 aims to answer to the following question: What to do with a $\mathcal{R}_{0}$ ? This will be addressed through four examples of mathematical and biological interest: the measure of epidemic risk, the "Herd immunity" as vaccination strategy, the assessment of the impact of biodiversity on the transmission of a trophically transmitted parasitic disease, and finally the study of disease persistence versus disease extinction. At last, Section 4 will focus on an example of a structured PDE model, due the incorporation of an infection load of the disease in the model, to illustrate the main difficulties that may arise when considering infinite dimensional epidemic systems.

\footnotetext{
* I am particularly grateful to Professor Gauthier Sallet for sharing documents he wrote related to a series of conferences he delivered on the topic. Some parts of this article have greatly benefited of these.

1 UMR 6249 Chrono-environnement - Université Bourgogne Franche-Comté, 16 route de Gray, F-25030 Besançon cedex FRANCE

(C) EDP Sciences, SMAI 2018
} 


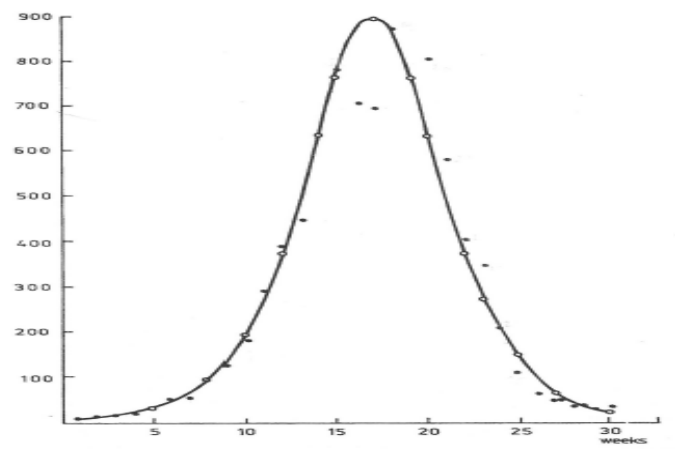

Figure 1. Number of plague deaths per time unit (week), from [16].

\section{From epidemiological definition of $\mathcal{R}_{0} \ldots$}

To introduce the concept of $\mathcal{R}_{0}$, let us start by a focus on the bubonic plague epidemic that affected Mumbai in 1905-1906: twenty years after this devasting epidemic, W. O. Kermack and A. G. McKendrick intended to answer to the following question: how can we prevent such an epidemic ? To achieve that goal, they built a dynamical model with the willingness to fit the collected data related to this epidemic. Their related work [16], published in 1927, shows one of the most known figures in mathematical epidemiology (see Figure 1).

To formulate the model they made several simplistic assumptions leading to the following considerations: they split the population of Mumbai into three categories, susceptible, infected and dead due to plague, and focused on the evolution of their densities, denoted $S(t)$ (susceptible), $I(t)$ (infected) and $R(t)$ (dead due to plague), according to some demographic and infectious processes. These considerations can be summarized through the scheme described in Figure 2, called epidemiological diagram.

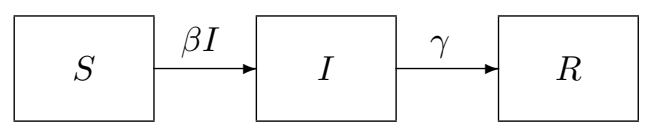

Figure 2. Epidemiological diagram of plague disease in Mumbai.

In the model, parameter $\beta>0$ denotes the transmission rate of the disease, so that the quantity $\beta I$ is the force of infection, and $\gamma>0$ is the mortality rate due to the disease. Kermack and McKendrick made a mathematical translation of the latter diagram by means of ODEs, and proposed the following model, so-called Kermack-McKendrick or SIR model, using a "SI" mass action law:

$$
\left\{\begin{array}{l}
\frac{d S(t)}{d t}=-\beta S(t) I(t) \\
\frac{d I(t)}{d t}=\beta S(t) I(t)-\gamma I(t) \\
\frac{d R(t)}{d t}=\gamma I(t)
\end{array}\right.
$$

The fundamental question, leading to the concept of $\mathcal{R}_{0}$, is the following: can we extract a tool, from system (1), to measure the disease risk and to prevent the apparition of an epidemic? This desired tool is the basic reprodution number $\mathcal{R}_{0}$. Several steps were necessary to formulate the epidemiological definition previously seen in introduction, i.e. the number of secondary infections resulting from a single primary infection into an otherwise susceptible population, and consequently to state a proper mathematical definition of $\mathcal{R}_{0}$. From the epidemiological definition, an intuitive sense shows easily that $\mathcal{R}_{0}$ has to be looked as a threshold. Indeed, 
following the introduction of a number $p$ of infected individuals into a susceptible population, then $\mathcal{R}_{0} p$ individuals will be infected after one stage, and more generally $\mathcal{R}_{0}^{k} p$ after $k$ stages. Consequently, $\mathcal{R}_{0}>1$ would imply a disease outbreak, while $\mathcal{R}_{0}<1$ its disappearance. Let us briefly remind the historical steps that led to a definition of $\mathcal{R}_{0}$ taking into account the notion of threshold and then allowed its mathematical translation. The interested reader can find in the article of J.A.P. Heesterbeek [13] more details about the history of the basic reproductive number $\mathcal{R}_{0}$.

At the early begining, this concept was introduced by R. Böckh in 1886 in a demographic purpose: R. Böckh tried to estimate the average number of female offspring produced by one female during her entire life. To do so, he calculated, from collected data in Berlin city during the year 1879, the product of the age-dependent survival probability with the fertility rate. Taking into account in his calculus the sex ratio, he got the estimation of 1.06, that can be considered as the first $\mathcal{R}_{0}$ value.

The second step was the mathematical formalization of the calculus made by R. Böckh. In 1925, L-J. Dublin and A. Lotka (also known for the formulation of a predator-prey model) proposed the first mathematical formula for a $\mathcal{R}_{0}$, linked to the age-dependent survival probability of women and to the fertility rate as follows:

$$
\mathcal{R}_{0}=\int_{0}^{\infty} \underbrace{\mathcal{P}(a)}_{\text {survival }} \underbrace{\beta(a)}_{\text {fertility }} d a
$$

One can note that, at this step, the latter definition of $\mathcal{R}_{0}$ is linked to demography but is not yet an epidemiological concept.

The link with epidemiology is due to R. Ross ${ }^{1}$ who published at the beginning of the 20th century a series of papers devoted to the study of the spread and control of malaria. He identified the anophelene mosquitoes as the vector for malaria transmission and developed a mathematical model that showed that malaria could be eradicated as long as the number of mosquitoes per human was brought below a threshold value (and so was for the corresponding $\mathcal{R}_{0}$ ). Although highly controversial at this time, this result was significant because it showed that it was not necessary to eliminate all the mosquitoes to eradicate malaria. This result, published by Ross in [30], is called "Ross' mosquitoe theorem", and is a phenomenon that has been generalized to many other controllable diseases, currently known as the "Herd immunity" effect (see Section 3 of the present article for a more detailed explanation).

Following the Ross' work on the specific case of malaria, Kermack and McKendrick then undertook to extend the threshold notion to communicable diseases, by developping through the publications [16-18] the well-known SIR model previously presented and the related "threshold theorem" (see the article [2] of R. M. Anderson for a review). The case of the 1905 plague epidemic in Mumbai is an example of such communicable disease on which they focused.

At this period (beginning of the 30's), the threshold notion is already introduced in mathematical epidemiology, but without making the link with demographics and the notion of reproduction formulation. This latter will finally be introduced in 1952 by G. McDonald, headmaster of the Ross institute, stating the following definition in [25] and using the symbol $z_{0}$ for the threshold:

\section{"Basic Reproduction Rate of malaria, Definition. The number of infections distributed in a community as the direct result of the presence in it of a single primary non-immune case."}

It's finally during the Dahlem conference of 1982, following a series of article published in the field during the 70's by applied mathematicians and ecologists (C.E.G. Smith, K. Dietz, H.W. Hethcote, R.M. Anderson, R.M. May, see $[1,9,14,32])$, that the terminology of basic reproduction rate, with notation $\mathcal{R}_{0}$ instead of $z_{0}$ resuming Lotka's formula (2), was adopted and linked to the following definition, as stated in introduction:

\footnotetext{
${ }^{1}$ Nobel prize of medicine in 1902 for his work on malaria and in particular the discovery of plasmodium falciparum, parasite at the origin of this disease
} 


\begin{abstract}
"Basic Reproduction Number, Epidemiological Definition. The number of secondary infections resulting from a single primary infection into an otherwise susceptible population."
\end{abstract}

A proper mathematical definition of $\mathcal{R}_{0}$ was finally introduced by O. Dikemann, J.A.P. Heersterbeek and J.A.J. Metz in 1990, linked to the dominant eigenvalue of the so-called "next-generation operator" (see [7, 8]). In this definition the authors focus on a specific steady state of the epidemiological dynamical system, commonly called the disease-free equilibrium (DFE), corresponding to the situation where the population is at an equilibirum that is characterized by a constant susceptible population in absence of the infectious agent. The $\mathcal{R}_{0}$ value, i.e. the dominant eigenvalue of the next generation operator, aims to ensure the stability $\left(\mathcal{R}_{0}<1\right)$ otherwise the instability $\left(\mathcal{R}_{0}>1\right)$ of the DFE. A more mathematically workable definition for the $\mathcal{R}_{0}$, when dealing with dynamical systems, is therefore:

\title{
"Basic Reproduction Number, Mathematical Definition. The number of new infections produced by a typical infective individual in a population at a DFE."
}

This latter definition has the interest to be valuable for ODEs as well as infinite dimensional dynamical systems, but appears more theoretical than pratical and so yields the following natural question: How can we concretely calculate a $\mathcal{R}_{0}$ value from a dynamical system modeling an epidemic process?

\section{2. ... TO MATHEMATiCAL CALCULATiON OF $\mathcal{R}_{0}$}

In this section, we present an efficient method for $\mathcal{R}_{0}$ calculation in a deterministic finite dimensional case. This method, proposed by P. Van Den Driessche and J. Watmough in [38], aims to make explicit the determination of the dominant eigenvalue of the "next-generation operator" introduced in [8] (see previous section) when regarding the specific case of ODEs compartmental epidemic models. Consequently to the finite dimension, it is called "next generation matrix" method, and is very useful to put into practice.

The authors propose to write the ODEs epidemic system by separating the state variables and the entering fluxes related to the infectious process from the others (see Figure 3). The dynamics is then written as the following compartmental system,

$$
\dot{x}(t)=f(x(t)), \quad x=(x_{1} \ldots, x_{p}, \underbrace{x_{p+1}, \ldots, x_{n}}_{\text {infected }})^{T}
$$

with the following partition of $f: \mathbb{R}^{n} \rightarrow \mathbb{R}^{n}$,

$$
f(x)=\mathcal{F}(x)+\underbrace{\mathcal{V}(x)}_{=\left(\mathcal{V}^{+}-\mathcal{V}^{-}\right)(x)}
$$

where $\mathcal{F}, \mathcal{V}^{+}$and $\mathcal{V}^{-}$are the nonnegative functions defined as follows:

- $\mathcal{F}_{i}$ flux of newly infected in compartment $i$,

- $\mathcal{V}_{i}^{+}$(resp. $\mathcal{V}_{i}^{-}$) other entering fluxes (resp. leaving fluxes) related to compartment $i$.

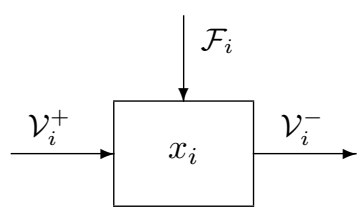

Figure 3. State variable, entering and leaving fluxes related to compartment $i$. 
With such a partition, the DFE is given by $x^{*}=\left(x_{1}^{*}, \ldots, x_{p}^{*}, 0, \ldots, 0\right)$ and one can prove that

$$
D_{x^{*}} \mathcal{F}=\left(\begin{array}{cc}
0 & 0 \\
0 & F
\end{array}\right), \quad D_{x^{*}} \mathcal{V}=\left(\begin{array}{cc}
J_{1} & J_{2} \\
0 & V
\end{array}\right)
$$

where $F$ is a positive matrix and $M$ a Metzler matrix, i.e a matrix in which all the off-diagonal components are nonnegative.

Let us denote, for any square matrix $Q$, by $S p(Q)$ the spectrum of $Q$, by $\rho(Q)=\max \{|\lambda|, \lambda \in S p(Q)\}$ the spectral radius of $Q$ and $\alpha(Q)=\max \{\operatorname{Re}(\lambda), \lambda \in S p(Q)\}$ the stability modulus of $Q$. Then by adapting the next-generation operator method, the authors in [38] state the following definition:

Definition 2.1. If $\alpha(V)<0$ then one defines the basic reproduction number linked to the DFE $x^{*}$ of system (3) as

$$
\mathcal{R}_{0}=\rho\left(-F V^{-1}\right)
$$

With such a definition, the following results hold:

\section{Theorem 2.2.}

(1) If $\mathcal{R}_{0}>1$ then the DFE $x^{*}$ in unstable.

(2) If $\mathcal{R}_{0}<1$, then the DFE $x^{*}$ is asymptotically stable under the condition $\alpha\left(J_{1}\right)<0$.

Sketch of proof - The main goal here is to make the link between $\mathcal{R}_{0}$ and the value of the real part of the dominant eigenvalue of $-F V^{-1}$. To achieve that goal, the proof lies on the three following ingredients (see Appendix): a weak version of the Perron-Frobenius theorem 4.1, the Lyapunov linearization theorem 4.3 and the Varga theorem 4.2.

Firstly, the theory about Metzler matrices allows to prove that $-F V^{-1} \geq 0$. This latter matrix being non-negative, the Perron-Frobenius theorem 4.1 implies that the spectral radius, i.e. $\mathcal{R}_{0}$, is an eigenvalue of the matrix. Then, linearizing the system in (3) about the DFE, the Poincaré-Lyapunov theorem 4.3 allows to consider the stability modulus of the differential in the sense where $\alpha\left(D_{x^{*}} f\right)>0$, resp. $\alpha\left(D_{x^{*}} f\right)<0$, implies the instability, resp. the stability, of $x^{*}$ for the nonlinear system $(3)$. Following the partition $f=\mathcal{F}+\mathcal{V}$, we focus on the sign of $\alpha(F+V)$ and $\alpha\left(J_{1}\right)$. One can note that, since $\alpha(V)<0$, the Varga theorem 4.2 implies that $\alpha(F+V)<0 \Leftrightarrow \mathcal{R}_{0}<1$ and by continuity, $\alpha(F+V) \leq 0 \Leftrightarrow \mathcal{R}_{0} \leq 1$. Consequently, we have:

1) $\mathcal{R}_{0}>1 \Rightarrow \alpha(F+V)>0 \Rightarrow \alpha\left(D_{x^{*}} f\right)>0$

2) $\mathcal{R}_{0}<1 \Rightarrow \alpha(F+V)<0$ and so $\alpha\left(D_{x^{*}} f\right)<0$ if $\alpha\left(F_{1}\right)<0$.

\section{Remark 2.3.}

(1) The definition 2.1 does not suppose that the DFE is unique. In the case of the existence of multiple DFEs, it can be possible to calculate a $\mathcal{R}_{0}$ value related to each equilibrium. But the definition of a global $\mathcal{R}_{0}$ is much more complicate, and requires a case-by-case study.

(2) The condition $\alpha(V)<0$ stated in Definition 2.1 ensures, due to Metzler matrices theory, the inversibility of $V$. This condition is often statisfied in practical situations.

(3) In the article [38], the authors assume in the definition of $\mathcal{R}_{0}$, that the DFE $x^{*}$ is asymptotically stable under the condition $\mathcal{F} \equiv 0$. This assumption clearly implies that $\alpha(V)<0$ and $\alpha\left(J_{1}\right)<0$, but is not necessary to define $\mathcal{R}_{0}$ as well as to obtain the instability of the DFE when $\mathcal{R}_{0}>0$, since the existence of an eingenvalue with positive real part is enough.

(4) Refering to the work of Diekmann et al. [8], $-F V^{-1}$ is called the next-generation matrix. This can be seen through the mathematical definition of $\mathcal{R}_{0}$ stated in Section 1: let us suppose that the system is at the DFE $x^{*}$ and let us determine the number of infected resulting from the introduction of an initially infected population $(0, \ldots, 0,1, \ldots, 1)=(\mathbf{0}, \mathbf{1}) \in \mathbb{R}^{n}$ into the susceptible one. Without considering the contamination process, i.e by supposing $\mathcal{F}=0$, then the population densities follow the dynamics $\dot{x}(t)=\left(D_{x^{*}} \mathcal{V}\right) x(t)$, so that at time $t, x(t)=\left(\mathbf{0}, e^{t V} \mathbf{1}\right)$ and the time-cumulative number of infected 
coming from this introduction is formally given by

$$
\int_{0}^{+\infty} e^{t V} \mathbf{1} d s=-V^{-1} \mathbf{1}
$$

Then taking into account the contamination process, $-F V^{-1} \mathbf{1}$ represent the quantity of new infected cases generated by the introduction of $\mathbf{1}$ infected individuals.

(5) An efficiency of this method lies in the fact that it is a dimensional reduction method, since the determination of the stability of modulus of $D_{x^{*}} f$, required to perform the stability analysis of $x^{*}$, is reduced to the determination of the spectral radius of the next-generation matrix $-F V^{-1}$ that is of lower dimension.

(6) The authors also investigate in [38] the specific case where $\mathcal{R}_{0}=1$, i.e. an analysis of the center manifold near $x^{*}$.

Example 2.4. To conclude this section, we focus on the SIR model (1). In this case, we can easily apply the next-generation matrix method to make explicit the basic reproduction number.

Note first that since the total population $S(t)+I(t)+R(t)$ is constant, one can focus only on the equation in $S$ and $I$. Clearly the partition is then $f=\mathcal{F}+\mathcal{V}^{+}-\mathcal{V}^{-}$where $\mathcal{F}\left(x_{1}, x_{2}\right)=\left(0, \beta x_{1} x_{2}\right), \mathcal{V}^{+}\left(x_{1}, x_{2}\right)=(0,0)$ and $\mathcal{V}^{-}\left(x_{1}, x_{2}\right)=\left(-\beta x_{1} x_{2},-\gamma x_{2}\right)$. When looking for a $D F E$, it is easy to check that every $\left(S^{*}, 0\right), S^{*} \geq 0$, is DFE for (1). From here, a simple calculation then gives $F=\beta S^{*}$ and $V=-\gamma$. The results of Section 2 finally show that

$$
\mathcal{R}_{0}=\frac{\beta S^{*}}{\gamma}
$$

\section{What TO DO WITH A $\mathcal{R}_{0}$ ?}

This question is addressed through four examples that illustrate the usefulness of the basic reproduction number in mathematical epidemiology.

\subsection{Example 1: a measure of epidemic risk}

The $\mathcal{R}_{0}$ can be used as a predictive tool to prevent a disease outbreak, the aim being to link a dynamic model with temporal collected data. By "to link", we mean the determination of values of the parameters involved in the model so that the solution of the dynamical system fits the data. For example, let us address such an issue for the SIR model (1) seen in Section 1, as Kermack and McKendrick intended to do. Denoting by $\left(S_{0}, I_{0}, R_{0}=0\right)$ the initial condition, the questions we raise are the following: Can we find suitable values of parameters $\left(\beta, \gamma, S_{0}, I_{0}\right) \in\left(\mathbb{R}_{+}\right)^{4}$ that allow

(1) to fit the solution of (1) with the collected data (as in Figure 1);

(2) to estimate the $\mathcal{R}_{0}$ value?

We recall that the data on which Kermack and McKendrick focused are time series related to the number of plague deaths per time unit, given in the model by the quantity $\frac{d R(t)}{d t}$. But in fact, they did not address the two previous raised questions in the article [16]. Indeed, the SIR system cannot be analytically integrated and numerical simulations were not possible at this time. They tackled the question of fitting by getting around this problem doing the following approximation of the number of death per time unit,

$$
\frac{d R(t)}{d t} \approx \frac{A}{\cosh ^{2}(B t-\phi)}
$$

where quantities $A, B$ and $\phi$ depend on the parameters involved in the model. With a fit "by hand" using such an approximation, the authors finally got the curve presented in Figure 1.

Although the simulations of the ODEs sytem (1) (see Figure 4) could make us believe that, for a high enough value of $\mathcal{R}_{0}$, the curve of number of death per time unit (proportional to the epidemic curve) could coincide 


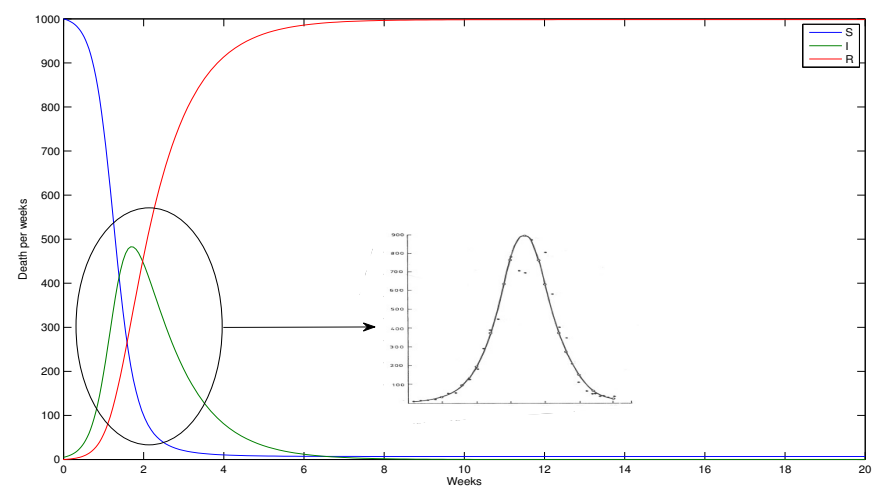

FiguRE 4. Curves of the SIR model for $\mathcal{R}_{0}=5$.

with the one in Figure 1 and consequently fit the data in a suitable way, N. Bacaër recenlty showed in [3] that fitting the epidemic curve under the assumption of constant parameters leads to unreasonable parameter and consequently unreasonable $\mathcal{R}_{0}$ values. In other words, the mathematical problem underlying the two questions raised above is ill-posed. Due to the simple modeling assumptions they made, this result was expected by Kermack and Mc Kendrick, as they noted: "None of these assumptions are strictly fulfilled and consequently the numerical equation can only be a very rough approximation. A close fit is not to be expected, and deductions as to the actual values of the various constants should not be drawn".

However, although if the latter example shows that the determination of a $\mathcal{R}_{0}$ value through a mathematical model fitting collected data is a challenging work, this approach is extensively used in practical situations to prevent outbreaks of current diseases, oftenly using the SIR model through several various versions. For example one can cite the IDEA model, a SIR discrete model that was developped in [11] and used to simulate time projections of the Ebola epidemics of 2014 in several countries of West Africa (see Figure 5). Antoher example is the stochastic SEIR model (with an "Exposed" class) developped by A. Flahault and his collaborators in 2009 in [12] to asses the H1N1 influenza pandemia risk. These models focus on the way to control the disease by using the "Herd immunity" effect as vaccination strategy.

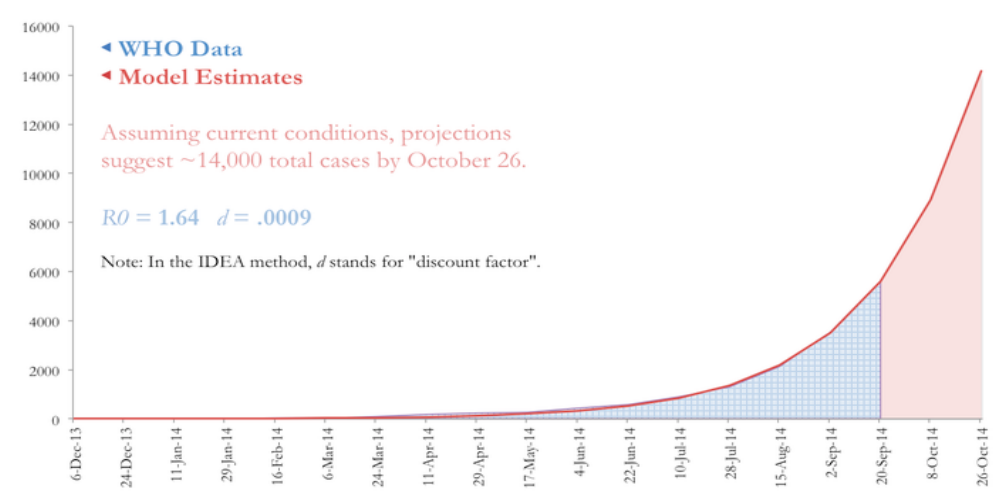

Figure 5. Ebola 2014: projections of the cumulative cases in West Africa, from healthmap.org. 


\subsection{Example 2: "Herd immunity" as vaccination strategy}

Herd immunity is a form of immunity that occurs when the vaccination of a significant portion of a population provides a measure of protection for individuals who have not developed immunity. As mentionned in Section 1, this concept was initially thought by R. Ross and his famous "Ross' mosquitoe theorem". To study the spread of malaria, he proposed in [30] the following model

$$
\left\{\begin{array}{l}
\frac{d I_{H}(t)}{d t}=a b_{1} I_{M} \frac{H-I_{H}}{H}-\gamma I_{H} \\
\frac{d I_{M}(t)}{d t}=a b_{2}\left(M-I_{M}\right) \frac{I_{M}}{M}-\mu I_{M}
\end{array}\right.
$$

with

- $M$ (resp. $H)$ constant population of mosquitoes (resp. humans)

- $I_{M}\left(\operatorname{resp} I_{H}\right)$ number of infected mosquitoes (resp. humans)

- a number of bites per mosquitoe and time unit

- $b_{1}$ probability that a bite generates a human infection

- $b_{2}$ probability that a mosquitoe becomes infected

- $1 / \gamma$ infection period for human

- $1 / \mu$ mosquitoe lifespan

The application of the next-generation matrix method gives, for the DFE $(0,0)$,

$$
\mathcal{R}_{0}=\sqrt{\frac{a^{2} b_{1} b_{2} M}{\gamma \mu H}}
$$

The square root in the latter equation is the consequence of the two-phases infectious process that occurs. Indeed, it requires two generations of infections, human to mosquitoe and mosquitoe to human, to consider the secondary infected humans from humans. The square root then represents the average in a geometric sense. Furthermore, one can check that the basic reproduction number $\mathcal{R}_{0}$ is a continuous decreasing function of the ratio of the population of mosquitoes per human $\frac{M}{H}$, so that a small enough value of $\frac{M}{H}$ guarantees that $\mathcal{R}_{0}<1$, as announced by Ross with the mosquitoe theorem. Since this first model about malaria spread was proposed by Ross, many others have been formulated proposing the calculation of $\mathcal{R}_{0}$ values, as presented in the article [33].

To enlighten the "Herd immunity" as vaccination strategy, let us focus on a specific example of epidemic model. Consider a disease with the following characteristics:

- no vertical transmission;

- exposure period;

- no native immunity;

- no possible recovery,

represented with the following epidemiological diagram:

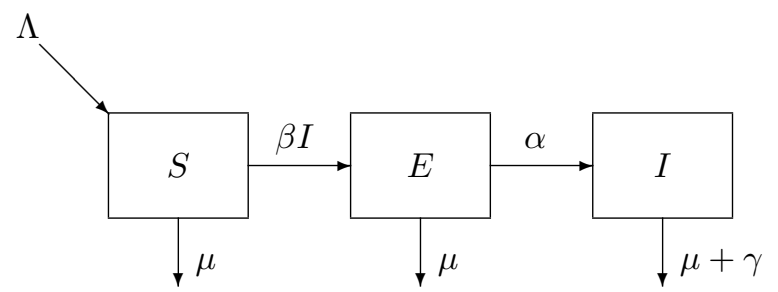

Figure 6. Epidemiological diagram of the SEI model. 
Such a SEI model is mathematically translated through the following ODEs system,

$$
\left\{\begin{array}{l}
\frac{d S(t)}{d t}=\Lambda-\beta S(t) I(t)-\mu S(t) \\
\frac{d E(t)}{d t}=\beta S(t) I(t)-(\alpha+\mu) E(t) \\
\frac{d I(t)}{d t}=\alpha E-(\gamma+\mu) I(t)
\end{array}\right.
$$

whose DFE $x^{*}$ and the basic reproduction number $\mathcal{R}_{0}$ are given by

$$
x^{*}=\left(\frac{\Lambda}{\mu}, 0,0\right) \quad ; \quad \mathcal{R}_{0}=\frac{\alpha \beta \Lambda}{\mu(\mu+\alpha)(\mu+\gamma)}
$$

The issue we ask, related to the "Herd immunity" effect, is the following: Which minimal proportion of the new borns should be vaccined to avoid the apparition of epidemics?

Denoting by $\epsilon \in(0,1)$ the proportion of vaccinated, the entering flux $\Lambda$ in the susceptible class then rewrites in the model with vaccination as $(1-\epsilon) \Lambda$, so that the basic reproduction number $\tilde{\mathcal{R}}_{0}$ satisfies

$$
\tilde{\mathcal{R}}_{0}=(1-\epsilon) \mathcal{R}_{0},
$$

and the vaccination of a proportion $\epsilon>1-\frac{1}{\mathcal{R}_{0}}$ would then imply that $\tilde{\mathcal{R}_{0}}<1$.

\begin{tabular}{lcc}
\hline \hline Disease & $\mathcal{R}_{0}$ & Herd immunity \\
\hline Mumps & $4-7$ & $75-86 \%$ \\
Polio & $5-7$ & $80-86 \%$ \\
Small pops & $5-7$ & $80-85 \%$ \\
Diphteria & $6-7$ & $85 \%$ \\
Rubella & $6-7$ & $83-85 \%$ \\
Pertussis & $12-17$ & $92-94 \%$ \\
Measles & $12-18$ & $83-94 \%$ \\
\hline \hline
\end{tabular}

TABLE $1 . \mathcal{R}_{0}$ and herd immunity thresholds for vaccine-preventable diseases, from [10].

The "Herd immunity" protection is extensively used all over the world for many cases of vaccinable diseases (see Table 1 and reference [10]). One can particularly cite the specific case of smallpox, that was eradicated in 1977 (officially annonced in 1980 by the World Health Assembly), following an historic global campaign of surveillance and vaccination, called the "Smallpox Eradication Programme", based on "Herd immunity" strategy.

\subsection{Example 3: impact of the biodiversity on a disease dynamics}

The third example on which we focus to enlighten the potential usefulness of the basic reprodution number deals with eco-epidemiological modeling of parasitic infections. The effects of biodiversity on the transmission of such zoonotic diseases recently became a major focus for the worldwide public health [21,39]. The first investigations of biodiversity effects have been investigated in 2000 (see [26]) for the Lyme disease, vectorized by ticks among mammals, birds and reptiles, where the dilution hypothesis, i.e that biodiversity could be protective against the pathogen transmission, was stated and explained by a difference in hosts' competence to accquire and to transmit the parasite.

Recently, a similar issue was raised about the parasitic disease echinococcosis. Emerging in Eastern Europe, Nothern Asia and America, it is caused by the tapeworm Echinococcus multilocularis (Em). To exist and 


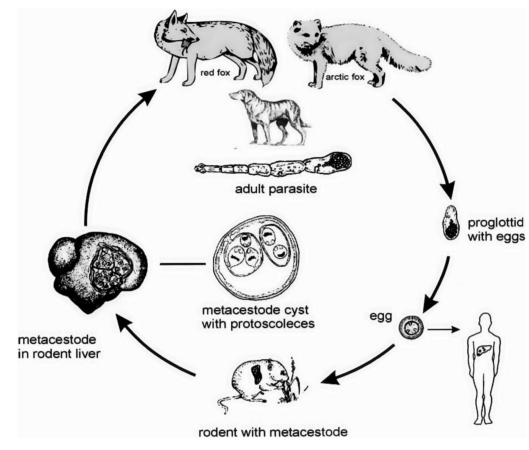

Figure 7. Echinococcus multilocularis cycle, from [37].

developp, this worm needs a specific cycle (see Figure 7): it is found in definitive hosts (DHs), such as foxes, dogs, coyotes and many other wild carnivores, and in intermediate hosts (IHs), mainly wild rodents. The DHs becomes infected by eating IHs and they then excrete the eggs in their faeces, these latter being a source of contamination for the IHs. Humans become infected by ingesting food (berries, herbs, mushrooms...) contaminated with DHs faeces or potentially due to a contact with an infected animal. The interested reader can find in [29] a review about the transmission of the parasite. Clearly, the evolution of the population of parasites Em strongly depends on the "quality" of the trophic links between the DHs, as predators, and the IHs, as preys. Therefore, to assess the persistance of the parasite, eco-epidemiologists wonder the following question: How the biodiversity of IHs, and more precisely the variability of their competence and of their proportion, may influence the dynamics of the parasite?

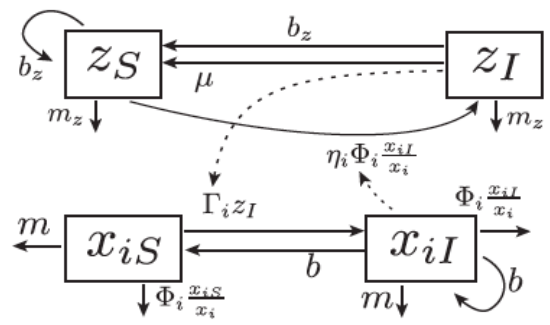

\begin{tabular}{cl}
\hline \hline Symbol & Parameter Definition \\
\hline$b, b_{z}$ & Birth rate of prey and predator \\
$m, m_{z}$ & Mortality rate of prey and predator \\
$k, k_{z}$ & Carrying capacity for prey and predator \\
$\mu$ & Recovery rate of the predator \\
$\eta_{i}$ & Probability that larvae from vole $i$ mature in a fox \\
$\Gamma_{i}$ & Rate of infection of voles (i.e. susceptibility) \\
$\eta_{i} \Gamma_{i}$ & Competence of prey to be infected and to transmit the parasite \\
$\Phi_{i}\left(x_{1}, x_{2}\right)$ & Ingestion rate of prey $i$ (Functional Response) \\
$\eta_{i} \Gamma_{i} \Phi_{i}\left(x_{1}, x_{2}\right)$ & Force of infection with prey $i$ \\
\hline \hline
\end{tabular}

Figure 8. Em transmission epidemiological diagram and parameters involved in the model, from [5].

This latter question has been tackled in [5] where the authors focused on the transmission of the parasite in Eastern Europe, where the DHs are the red foxes (Vulpes vulpes) and the IHs are two small mammals, the common vole (Microtus arvalis) and the water vole (Arvicola scherman). Following a preliminary ecological modeling of the trophic relationships in [4], where it was shown that the property of prey switching, i.e a change 
in the preference of the predator to a prey species with a variation of its relative density, was a characteristic of the studied predator-prey interactions, the transmission of the $E m$ parasite has then been described using the epidemiological diagram of Figure 8 (where $i=1,2$ denotes a type of prey, $S$, resp $I$, refers to suceptible populations, resp. infected), and translated through the ODEs system (4).

$$
\begin{aligned}
& \text { growth } \\
& \frac{d z_{S}}{d t}=b_{z} z-\left(m_{z}+\left(b_{z}-m_{z}\right) \frac{z_{S}+z_{I}}{k_{z}}\right) z_{S} \\
& \text { predation } \\
& \text { epidemic } \\
& \frac{d x_{i S}}{d t}=b x_{i}-\left(m+(b-m) \frac{\sum_{j} x_{j S}+x_{j I}}{k}\right) x_{i S}-\Phi_{i}\left(x_{1}, x_{2}\right) \frac{x_{i S}}{x_{i}} z-\quad z_{I} \Gamma_{i} x_{i S} \\
& \frac{d z_{I}}{d t}=-\left(m_{z}+\left(b_{z}-m_{z}\right) \frac{z_{S}+z_{I}}{k_{z}}\right) z_{I} \quad+z_{S} \sum_{i} \eta_{i} \Phi_{i}\left(x_{1}, x_{2}\right) \frac{x_{i I}}{x_{i}}-\mu z_{I} \\
& \frac{d x_{i I}}{d t}=-\left(m+(b-m) \frac{\sum_{j} x_{j S}+x_{j I}}{k}\right) x_{i I}-\Phi_{i}\left(x_{1}, x_{2}\right) \frac{x_{i I}}{x_{i}} z+\quad z_{I} \Gamma_{i} x_{i S}
\end{aligned}
$$

Contrary to the previous epidemic models presented in the article, where the nonlinear part was quadratic due to the "SI" mass action law, the latter model presents a more complex one, since the disease transmission is carried out through the trophic interactions, involving the nonlinear shape $\Phi_{i}$. Such a function, called functional response, is a modeling characteristics of predator-prey interactions that represents the intake rate of the predator as a function of prey densities. The most common shapes of functional responses are Holling type I (linear), Holling type II (first order rational) and Holling type III (second order rational), see [4, 15, 35] for a review about all the existing current shapes.

For the system (4), the following result was proved in [5]:

Theorem 3.1. If $\Phi_{i}$ satisfy the property of prey switching, then their exists a coexisting DFE $\left(z^{*}, x_{1}^{*}, x_{2}^{*}, 0,0,0\right)$ with the following associated basic reproduction number:

$$
\mathcal{R}_{0}=\sqrt{\frac{z^{*}}{b\left(b_{z}+\mu\right)} \times\left(\eta_{2} \Gamma_{2} \Phi_{2}\left(x_{1}^{*}, x_{2}^{*}\right)+\eta_{1} \Gamma_{1} \Phi_{1}\left(x_{1}^{*}, x_{2}^{*}\right)\right)}
$$

Sketch of proof - It lies into three steps:

(1) a model reduction with different time scales (parasite cycle VS. host dynamics);

(2) the change of variables $\left(x_{1}, x_{2}\right) \mapsto\left(x_{1}+x_{2}, \frac{x_{1}}{x_{1}+x_{2}}\right)$ to get the exisence of DFE;

(3) the application of the next generation matrix to the reduced model.

The question initially asked, i.e the assesment of the impact of the biodiversity on the dynamics of the parasite, finds answer through the parameters $\Gamma_{i}$ (for the IHs' competences) and the quantities $\lambda_{i}^{*}=\frac{x_{i}^{*}}{x_{1}^{*}+x_{2}^{*}}($ for the IHs' proportions). Figure 9 presents the influence of biodiversity on the $\mathcal{R}_{0}$ when species 1 is less competent than species 2 (i.e $\Gamma_{1}<\Gamma_{2}$ ), for three different functional responses. We observe for each case that $\mathcal{R}_{0}$ decreases when the proportion of the less susceptible prey (here $\lambda_{1}^{*}$ ) increases. The dilution effect is represented by an increase of biodiversity from $\lambda_{1}^{*}=0$ to 0.5 , while the amplification is observed with an increase of biodiversity from $\lambda_{1}^{*}=1$ to 0.5 . Finally, one can note that the characteristics of predation impact the disease tranmsission: 
if the dilution and amplification velocities increase with respect to the total amount of preys for each functional response, this phenomenon is intensified with Holling type II.
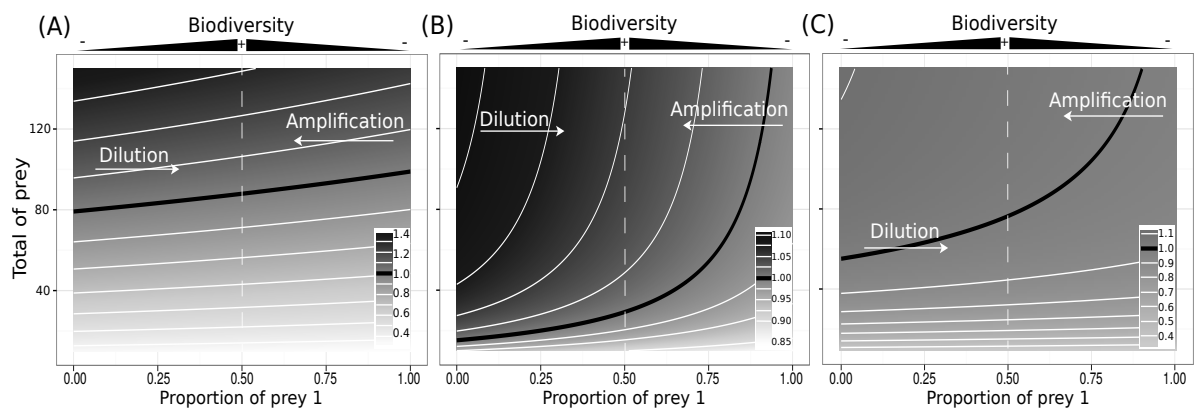

FiguRe 9. Influence on the basic reproductive number of the biodiversity in prey for different competences, with functional reponse Holling I (left), II (middle) and III (right), from [5]

\subsection{Example 4: Extinction VS. persistence study}

A mathematical interesting issue related to the basic reproduction number lies in the study of the time symptotic properties of the solutions of the epidemmic dynamical systems. Indeed, the next generation matrix ensures that, for the ODEs systems and under suitable assumptions (see Section 2), the DFE is locally asymptotically stable when $\mathcal{R}_{0}<1$. But what could be the behavior of the infected curve when $\mathcal{R}_{0}>1$, would it coincide with a disease persistence ?

To illustrate such a study, let us focus on a concrete example, given by the following generic case of "SI" model:

$$
\left\{\begin{array}{l}
\frac{d S(t)}{d t}=\Lambda-\beta S(t) I(t)-\mu_{S} S(t) \\
\frac{d I(t)}{d t}=\beta S(t) I(t)-\mu_{I} I(t)
\end{array}\right.
$$

For system (5), the DFE is given by $\left(S^{*}, 0\right)=\left(\frac{\Lambda}{\mu_{S}}, 0\right)$ and $\mathcal{R}_{0}=\frac{\beta \Lambda}{\mu_{S} \mu_{I}}$. The next-generation matrix implies that the DFE is unstable when $\mathcal{R}_{0}>1$. Is it sufficient to conclude that the disease is persistent ? The answer is negative, since the existence of a nonnegative initial condition for which the associated solution would satisfy $\liminf _{t \rightarrow+\infty}\left\|(S(t), I(t))-\left(S^{*}, 0\right)\right\|=0$ is not in contradiction with the instability of the DFE. We therefore need to state a stronger definition of persistence.

Definition 3.2. The disease is persistent if

$$
\exists \varepsilon>0, I_{0}>0 \Rightarrow \liminf _{t \rightarrow+\infty} I(t) \geq \varepsilon
$$

In other words, the persistence means that the infected population remains over time, with a minimal threshold that is uniform in the initial condition. A way to study if a disease is persistent is to investigate the existence of a globally stable endemic equilibrium (EE). In the case of system (5), their is a unique EE, given by $(\bar{S}, \bar{I})=\left(\frac{\mu_{I}}{\beta}, \frac{\mu_{S}}{\beta}\left(\mathcal{R}_{0}-1\right)\right)$ that exists only under the hypothesis $\mathcal{R}_{0}>1$ to ensure a biological reality. The following result holds, implying the disease persistence when $\mathcal{R}_{0}>1$ :

\section{Theorem 3.3.}

(1) If $\mathcal{R}_{0} \leq 1$ then the DFE is globally stable in $\left(\mathbb{R}_{+}\right)^{2}$;

(2) If $\mathcal{R}_{0}>1 \Rightarrow$ then the DFE is unstable and the EE is globally stable in the positive orthant $\mathcal{O} \subset \mathbb{R}^{2}$. 
Sketch of proof - Let us prove the persistent cas, i.e. the global stability of the EE when $\mathcal{R}_{0}>1$. We consider the following function:

$$
L(S, I)=\bar{S} g\left(\frac{S}{\bar{S}}\right)+\bar{I} g\left(\frac{I}{\bar{I}}\right)
$$

where $\bar{S}$ and $\bar{I}$ are the components of the EE and $g(x)=x-\ln x-1$ for $x>0$. The goal is to prove that function $L$ is a Lyapunov function on $\mathcal{O}$, i.e satisfies:

- $L(\bar{S}, \bar{I})=0$,

- $L$ is definite positive: $L(S, I)>0$ for $(S, I) \in \mathcal{O} \backslash\{(\bar{S}, \bar{I})\}$;

- $L$ is proper: $\|(S, I)\| \rightarrow \infty \Rightarrow L(S, I) \rightarrow \infty$;

- $\frac{d[L(S(t), I(t))]}{d t} \leq 0$.

If these latter points are satisfied, then the Lyapunov theorem implies the local asymptotic stability of the EE. The main issue is to prove the last point, the other being trivial or easy to check. Note that, since the EE is solution of (5), then the following equalities hold,

$$
\begin{gathered}
\Lambda=\mu_{I} \bar{I}+\mu_{S} \bar{S} \\
\beta \bar{S} I(t)=\mu_{I} I(t) \\
\beta S \bar{I}(t)=\frac{\Lambda S(t)}{\bar{S}}-\mu_{S} S(t)
\end{gathered}
$$

Then a differentiation gives

$$
\begin{aligned}
\frac{d[L(S(t), I(t))]}{d t} & =\Lambda-\mu_{S} S(t)-\Lambda \frac{\bar{S}}{S(t)}+\beta \bar{S} I(t)+\mu_{S} \bar{S}-\mu I(t)-\beta S(t) \bar{I}+\mu_{I} \bar{I} \\
& =\Lambda\left(2-\frac{\bar{S}}{S(t)}-\frac{S(t)}{\bar{S}}\right) \\
& =-\frac{\Lambda \bar{S}}{S(t)}\left(1-\frac{S(t)}{\bar{S}}\right)^{2} \leq 0
\end{aligned}
$$

and so the EE is asymptotically stable. We now prove the global attractiveness property of the EE. Denoting, using an abuse of notations, by $\dot{L}:(S, I) \in \mathcal{O} \mapsto \dot{L}(S, I)$ the functional in (7), it is then clear that the equality $\dot{L}(S, I)=0$ holds only on the straight line $S=\bar{S}$ of $\mathcal{O}$. Since the EE $(\bar{S}, \bar{I})$ is the only invariant set of system (5) on the straight line $S=\bar{S}$, then the Lasalle invariance principle (see [31]) implies the global attractiveness of $(\bar{S}, \bar{I})$ which, together with the asymptotic stability of the EE previously proved, show that the EE is globally stable with respect to $\mathcal{O}$.

When supposing that $\mathcal{R}_{0} \leq 1$, the results can be proved using a similar sketch of proof with the following function defined on $\tilde{\mathcal{O}}=\left\{(S, I) \in\left(\mathbb{R}_{+}\right)^{2}, S>0\right\}$ :

$$
V(S, I)=S^{*} g\left(\frac{S}{S^{*}}\right)+I
$$

We can prove that on $\tilde{\mathcal{O}}$,

$$
\dot{V}(S, I)=-\frac{\Lambda S^{*}}{S}\left(1-\frac{S}{S^{*}}\right)^{2}-\mu_{I}\left(1-\mathcal{R}_{0}\right) I \leq 0,
$$

ensuring the global attractiveness in $\tilde{\mathcal{O}}$, and finally in $\left(\mathbb{R}_{+}\right)^{2}$ since $S(t) \geq \frac{\Lambda}{\mu_{S}}>0$ from (5).

We can find in the litterature several articles in mathematical epidemiology that investigate the question of disease extinction VS. disease persistence for ODEs systems, by means of global analysis. One can in particular mention the case of SIR, SIRS and SIS models in $[19,20]$ and an extension to multi-strains SIR and SIS models in [6]. 


\section{A structured epidemic PDE MOdel}

In the last decades, some epidemic models incorporating, adding to time, another continuous variable have been proposed to discriminate the individuals of the infective population. One can particularly mention the structures of age of infection, infection load, immunity level, time remaining before disease detection (see [7], [22], [23], [24], [28], [34], [36] and references therein) with broad range of applications: nosocomial infections, HIV, salmonella, Bovine Spongiform Encephalopathy, Scrapie, Chronic Wasting Disease, Influenza...

These models, called structured population epidemic models, are described by systems of transport PDEs. Let us focus on the case of an infection load structure. Denoting by $i \in J=\left(i_{0},+\infty\right)$ the infection load in the infective class, it is supposed that the following evolution equation is satisfied $\frac{d i}{d t}=\gamma(i)$, where function $\gamma \in C^{1}(J, \mathbb{R})$ is positive on $J$ represents the growth velocity of the infection. It has been proposed in [28] a generalized ODEs model of SI type that incorporates the infection load structure:

$$
\left\{\begin{array}{l}
\frac{d S(t)}{d t}=\gamma-\mu_{0} S(t)-S(t) \int_{i_{0}}^{+\infty} \beta(i) I(t, i) d i \\
\frac{\partial I(t, i)}{\partial t}=-\frac{\partial(\gamma(i) I)(t, i)}{\partial i}-\mu(i) I(t, i)+\Phi(i) S(t) \int_{i_{0}}^{+\infty} \beta(i) I(t, i) d i \\
\gamma\left(i_{0}\right) I\left(t, i_{0}\right)=0, \\
S(0)=S_{0} \in \mathbb{R}_{+}, \quad I(0, \cdot)=I_{0} \in L_{+}^{1}(J)
\end{array}\right.
$$

The parameters and their definition are referenced in Table 2 . The boundary condition $\gamma\left(i_{0}\right) I\left(t, i_{0}\right)=0$ of $(8)$ represents an enterring flux. Indeed, it results from the conservation law $\partial_{t} I(t, i)=-\partial_{i}(\gamma(i) I)(t, i)$, a formal integration showing that

$$
\frac{d}{d t}\left(\int_{i^{-}}^{i} I(t, i)\right)=\underbrace{\gamma\left(i_{0}\right) I\left(t, i_{0}\right)}_{\text {entering flux in } i_{0} \text { entering flux in } i} \underbrace{-\gamma(i) I(t, i)} .
$$

\begin{tabular}{lc}
\hline \hline Parameter definition & symbol \\
\hline \hline recruitment flux & $\gamma$ \\
minimal infection load & $i_{0}$ \\
basic mortality rate & $\mu_{0}$ \\
disease mortality rate & $\mu$ \\
horizontal transmission rate & $\beta$ \\
infection load velocity & $\nu$ \\
infection load distribution at contamination & $\Phi$ \\
\hline \hline
\end{tabular}

TABLE 2. Parameters involved in the model.

The structure variable $i \in J$ involved in the PDE system (8) implies to deal with infinite dimensional systems. In such a case, many results that were previously stated and used in the case of ODEs models fail, or at least become more complicate and technical to investigate. In particular, this would imply the following major complications:

- it shall be ensured that a suitable theoretical framework (i.e the functional spaces) is defined;

- we have to deal with differential operators and anymore matrices;

- the spectral properties are different, since the spectrum of the differential operators may contain other elements than eigenvalues (such as essential spectrum);

- the analytical expression of $\mathcal{R}_{0}$ depends on the structure variable and the equilibria are functions of this latter; 
- the local stability properties through linearization and the Varga theorem fail (see theorem 4.3 and 4.2 in appendix);

- If the Lasalle invariance principle for the global attractiveness still holds in infinite dimension, the stability property ensured by Lyapunov functions fails.

However, these latter complications are of mathematical interest and often imply challenging but interesting works. Several positive results concerning the formulation of the basic reprodution number can be found in the litterature, mainly dealing with age of infection structure.

In the case of model (8), it has been proved in [28] that for the case of exponential growth diseases, i.e $\gamma(i)=\nu i$ where $\nu>0$, the basic reproduction number is given by

$$
\mathcal{R}_{0}=\frac{\gamma}{\mu_{0}} \int_{i_{0}}^{+\infty} \frac{1}{\nu i} \int_{i_{0}}^{i} \Phi(s) e^{-\int_{s}^{i} \frac{\mu(l)}{\nu l} d l} d s
$$

and is a sharp threshold for the stability property of the DFE and EE equilibria. In the more recent work [27], a global analysis was perfomed using infinite dimensionak Lyapunov functions generalizing the result of Theorem 3.3, providing the disease persistence result for system (8) when $\mathcal{R}_{0}>1$.

\section{APPENDIX}

Theorem 4.1 (A weak Perron-Frobenius theorem). If $A$ is a nonnegative matrix then $\rho(A)$ is an eingenvalue of $A$.

Theorem 4.2 (Varga theorem). For every Metzler matrix $A$, if $A=F+V$ with $F$ nonnegative and $\alpha(V)<0$, then $\alpha(A)<0 \Leftrightarrow \rho\left(-F V^{-1}\right)<1$.

Theorem 4.3 (Poincaré-Lyapunov linearization theorem). Let $x^{*}$ be a steady state of $\dot{x}(t)=f(x(t))$, where $f: \mathbb{R}^{n} \rightarrow \mathbb{R}^{n}$ is locally lpschitz continuous. Then $x^{*}$ is asymptotically stable if $\alpha\left(D_{x^{*}} f\right)<0$ and unstable if $\alpha\left(D_{x^{*}} f\right)>0$.

\section{REFERENCES}

[1] R. Anderson and R. M. (eds), Population Biology of Infectious Diseases, Springer-Verlag, 1982.

[2] R. Anderson And R. May, Infectious Diseases of Humans: Dynamics and control, Oxford University Press, 1991.

[3] N. BACAËR, The model of Kermack and McKendrick for the plague epidemic in Bombay and the type reproduction number with seasonality, J. Math. Biol., 64 (2012), pp. 403-422.

[4] V. Baudrot, A. Perasso, C. Fritsh, P. Giraudoux, and F. Raoul, The adaptation of generalist predators' diet in a multi-prey context : insights from new functional responses, Ecology, 97 (2016), pp. 1832-1841.

[5] V. Baudrot, A. Perasso, C. Fritsh, and F. Raoul, Competence of hosts and complex foraging behavior are two cornerstones in the dynamics of trophically transmitted parasites, J. Theor. Biol., 397 (2016), pp. 158-168.

[6] D. Bichara, A. IgGidr, And G. Sallet, Global analysis of multi-strains SIS, SIR and MSIR epidemic models, J. Appl. Math. Comput., 44 (2014), pp. 273-292.

[7] O. Diekmann and J. A. P. Heesterbeek, Mathematical Epidemiology of Infectious Diseases, Wiley Series in Mathematical and Computational Biology, John Wiley \& Sons, 2000.

[8] O. Diekmann, J. A. P. Heesterbeek, and J. A. J. Metz, On the definition and the computation of the basic reproduction ratio $R_{0}$ in models for infectious diseases in heterogeneous populations, J. Math. Biol., 28 (1990), pp. 365-382.

[9] K. DiETz, transmission and control of arbovirus diseases, in Epidemiology, Utah Conference Proceedings, SIAM, Philadelphia, 1975, pp. 104-121.

[10] M. Doherty, P. Buchy, B. Standaert, C. Giaquinto, and D. Prado-Cohrs, Vaccine impact: Benefits for human health, Vaccine, 34 (2016), pp. 6707-6714.

[11] D. Fisman, T. Hauck, A. Tuite, And A. Greer, An IDEA for short term outbreak projection: Nearcasting using the basic reproduction number, PLoS One, 8 (2013), p. e83622.

[12] A. Flahault, E. Vergu, And P.-Y. Bö̈Lle, Potential for a global dynamic of influenza a (h1n1), BMC Infect. Dis., 9 (2009), p. 129.

[13] J. Heesterbeek, A brief history of $r_{0}$ and a recipee for its calculation, Acta Biotheor., 50 (2002), pp. 189-204. 
[14] H. Нетнсоте, Mathematical models for the spread of infectious diseases, in Epidemiology, Utah Conference Proceedings, SIAM, Philadelphia, 1975, pp. 122-131.

[15] J. Jeschke, M. Kopp, And R. TollRian, Predator functional responses: discriminating between handling and digesting prey, Ecol. Monogr., 72 (2002), pp. 95-112.

[16] W. O. Kermack and A. G. McKendrick, A contribution to the mathematical theory of epidemics, Proc. R. Soc. Lond. Ser. A, 115 (1927), pp. 700-721.

[17] - Contributions to the mathematical theory of epidemics: 2, Proc. R. Soc. Lond. Ser. B, 138 (1932), pp. 55-83.

[18] - Contributions to the mathematical theory of epidemics: 3, Proc. R. Soc. Lond. Ser. B, 141 (1933), pp. 94-112.

[19] A. Korobeinikov, Lyapunov functions and global stability for SIR and SIRS epidemiological models with non-linear transmission, Bull. Math. Biol., 68 (2006), pp. 615-626.

[20] A. Korobeinikov And G. C. WAKe, Lyapunov functions and global stability for SIR, SIRS, and SIS epidemiological models, Appl. Math. Lett., 15 (2002), pp. 955-960.

[21] K. Lafferty, A. Dobson, And A. Kuris, Parasites dominate food web links, Proc. Natl. Acad. Sci. (PNAS), 103 (2006), pp. 11211-11216.

[22] B. Laroche and A. Perasso, Threshold behaviour of a SI epidemiological model with two structuring variables, J. Evol. Equ., 16 (2016), pp. 293-315.

[23] P. Magal and C. McCluskey, Two group infection age model: an application to nosocomial infection, SIAM J. Appl. Math., 73 (2013), pp. 1058-1095.

[24] P. Magal, C. McCluskey, and G. WebB, Lyapunov functional and global asymptotic stability for an infection-age model, Appl. Anal., 89 (2010), pp. 1109-1140.

[25] G. MCDonald, The analysis of equilibrium in malaria, Trop. Diseases Bull., 49 (1952), pp. 813-829.

[26] R. Ostfeld And F. Keesing, Biodiversity series: the function of biodiversity in the ecology of vector-borne zoonotic diseases, Can. J. Zool., 78 (2000), pp. 2061-2078.

[27] A. Perasso, Global stability and uniform persistence for an infection load-structured si model with exponential velocity, Submitted to Comm. Pure Appl. Anal., (2017).

[28] A. Perasso and U. Razafison, Asymptotic behavior and numerical simulations for an infection load-structured epidemiological models ; application to the transmission of prion pathologies, SIAM J. Appl. Math., 74 (2014), pp. 1571-1597.

[29] F. Raoul, D. Hegglin, And P. Giraudoux, Trophic ecology, behaviour and host population dynamics in Echinococcus multilocularis transmission, Vet. Parasitol., 213 (2015), pp. 162-171.

[30] R. Ross, The prevention of malaria, 2nd edition, John Murray, 1911.

[31] J. L. Salle and S. Lefschetz, Stability by Liapunov's Direct Method, Academic Press, New York, 1961.

[32] C. Sмiтh, Factors in the transmission of virus infections from animal to man, in Scientific Basis of Medicine, B. P. M. Federation, ed., Annual Reviews, Athlone Press, London, 1964, pp. 125-150.

[33] D. Smith, F. McKenzie, R. Snow, And S. Hay, Revisiting the basic reproductive number for malaria and its implications for malaria control, PLoS Biol., 5 (2007), pp. 531-542.

[34] H. Smith And H. Thieme, Dynamical systems and population persistence, Graduate Studies in Mathematics 118, American Mathematical Society, 2011.

[35] S. Smout, C. Asseburg, J. Matthiopoulos, C. Fernández, S. Redpath, S. Thirgood, and J. Harwood, The functional response of a generalist predator, PLoS ONE, 5 (2010), p. e10761.

[36] H. Thieme and C. Castillo-Chavez, How may infection-age-dependent infectivity affect the dynamics of hiv/aids ?, SIAM J. Appl. Math., 53 (1993), pp. 1447-1479.

[37] P. Torgerson, K. Keller, M. Magnotta, and N. Ragland, The global burden of alveolar echinococcosis, PLoS Negl. Trop. Dis., 4 (2010), p. e722.

[38] P. Van den Driessche and J. Watmough, Reproduction numbers and sub-threshold endemic equilibria for compartmental models of disease transmission, Math. Biosci., 180 (2002), pp. 29-48. John A. Jacquez memorial volume.

[39] C. Wood AND K. LAFFerTy, Biodiversity and disease: a synthesis of ecological perspectives on lyme disease transmission, Trends Ecol. Evol., 28 (2013), pp. 239-247. 